\title{
Knowledge-based IMRT planning for individual liver cancer patients using a novel specific model
}

\author{
Gang $\mathrm{Yu}^{1 \dagger}$, Yang $\mathrm{Li}^{1 \dagger}$, Ziwei Feng ${ }^{1}$, Cheng Tao ${ }^{2}$, Zuyi Yu', Baosheng $\mathrm{Li}^{2^{*}}$ and Dengwang $\mathrm{Li}^{\mathrm{1}^{*}}$
}

\begin{abstract}
Background: The purpose of this work is to benchmark RapidPlan against clinical plans for liver Intensity-modulated radiotherapy (IMRT) treatment of patients with special anatomical characteristics, and to investigate the prediction capability of the general model (Model-G) versus our specific model (Model-S).

Methods: A library consisting of 60 liver cancer patients with IMRT planning was used to set up two models (Model-S, Model-G), using the RapidPlan knowledge-based planning system. Model-S consisted of 30 patients with special anatomical characteristics where the distance from planning target volume (PTV) to the right kidney was less than three centimeters and Model-G was configurated using all 60 patients in this library. Knowledge-based IMRT plans were created for the evaluation group formed of 13 patients similar to those included in Model-S by Model-G, Model-S and manually (M), named RPG-plans, RPS-plans and M-plans, respectively. The differences in the dose-volume histograms (DVHs) were compared, not only between RP-plans and their respective M-plans, but also between RPG-plans and RPS-plans.

Results: For all 13 patients, RapidPlan could automatically produce clinically acceptable plans. Comparing RP-plans to M-plans, RP-plans improved $V_{95 \%}$ of PTV and had greater dose sparing in the right kidney. For the normal liver, RPG-plans delivered similar doses, while RPS-plans delivered a higher dose than M-plans. With respect to RapidPlan models, RPS-plans had better conformity index $(\mathrm{Cl})$ values and delivered lower doses to the right kidney $\mathrm{V}_{20 G y}$ and maximizing point doses to spinal cord, while delivering higher doses to the normal liver.
\end{abstract}

Conclusion: The study shows that RapidPlan can create high-quality plans, and our specific model can improve the $\mathrm{Cl}$ of PTV, resulting in more sparing of OAR in IMRT for individual liver cancer patients.

Keywords: Specific model, Knowledge-based planning, IMRT, Liver cancer

\section{Background}

Liver cancer is the fifth most common type of cancer and the third leading cause of cancer-related death worldwide. Consequently, liver cancer is an issue that needs to be urgently addressed [1]. Intensity-modulated radiotherapy (IMRT), based on computerized treatment plan optimization, permits the delivery of higher therapeutic

\footnotetext{
*Correspondence: 857317673@qq.com; lidengwang@sdnu.edu.cn

${ }^{\dagger}$ Equal contributors

2Shandong Medical Imaging and Radiotherapy Engineering Research Center, Department of Radiation Oncology, Shandong Cancer Hospital, Jinan 250014, People's Republic of China

${ }^{1}$ Shandong Province Key Laboratory of Medical Physics and Image Processing Technology, School of Physics and Electronics, Shandong Normal University, No.88, Wenhua East Road, Lixia District, Jinan, Shandong 250014, China
}

(c) The Author(s). 2018 Open Access This article is distributed under the terms of the Creative Commons Attribution 4.0 International License (http://creativecommons.org/licenses/by/4.0/), which permits unrestricted use, distribution, and reproduction in any medium, provided you give appropriate credit to the original author(s) and the source, provide a link to the Creative Commons license, and indicate if changes were made. The Creative Commons Public Domain Dedication waiver (http://creativecommons.org/publicdomain/zero/1.0/) applies to the data made available in this article, unless otherwise stated. adjacent normal tissue [2]. However, each step in the clinical workflow, from contouring to delivery, contains variability and uncertainties that ultimately translate into inconsistencies and inefficiency [3, 4].

A variety of solutions were studied, to reduce the variability present the back and forth between plan creation and approval [5-10]. Among these methods, knowledgebased planning is the most commonly used. It can predict achievable planning target volume (PTV) and DVHs for organs-at-risk (OARs) for prospective patients, utilizing models built using a library consisting of previous plans [11-19]. Chanyavanich et al. [18] used an algorithm based on mutual information to identify similar patients, and 
then generate new plans for the target cases. Zhu et al. [20] developed an evaluation tool for quantification, that generates DVHs based on organ volumes, as well as distance-to-target histograms (DTH), using a machine learning approach. Wu et al. [16] predicted DVHs of target cases by establishing the overlap volume histogram $(\mathrm{OVH})$. Based on that, Varian developed the Geometrybased Expected Dose (GED) algorithm and build a commercially available knowledge-based planning solution (RapidPlan, Varian Medical Systems, Palo Alto, CA), which semi-automates the treatment planning process. It uses a library of previous plans to build a model that can achieve OAR DVHs prediction range for a new patient, and subsequently guides the volumetric modulated arc therapy (VMAT) or IMRT optimization process using the Eclipse treatment planning system, along the inferior boundary of the DVH-prediction range. Previous work suggested that RapidPlan achieved clinically acceptable plans for different treatment sites [21-27]. To verify whether a model is suboptimal, Hussein et al. [24] suggested that there should be an insignificant effect on resulting plan quality when removing dosimetric outliers from the model training set. Similarly, Delaney et al. [28] found that statistical outliers removed from or added to models (5-10 outliers) had only a marginal impact on plan results. In a study by Tol et al. [27], a model created using 30 plans generated plans that were similar to those in a model based on 60 plans, when their plans were selected arbitrarily from all of 90 plans.

In this study, two models were developed, one general model and one specific model. All the studies mentioned above are based on the general model, which suits a wide variety of patient cases. However, the prediction ability of specific models tailored for specific patient cases, with specific anatomical features, has not been investigated. The aim of this study was (1) to evaluate the accuracy of RapidPlan prediction capability in IMRT (Eclipse, Varian) plans for individual liver cancer patients by using model libraries consisting of different total number of plans, with different similarity; and (2) to investigate the prediction capability of the general model vs. our specific model.

\section{Methods}

\section{Clinical plans}

Liver cancer patients were treated with IMRT from 2015 to 2016, planned based on the Eclipse treatment planning system. For all patients, the IMRT plans were created using 5 non-uniformly distributed coplanar fields $\left(0^{\circ}, 200^{\circ}\right.$, $240^{\circ}, 280^{\circ}, 320^{\circ}$ ) with the same photon beam setting of DVO, including number and beams energy. The prescribed dose was set as 50 Gy in 25 fractions. All plans were normalized to a mean dose of PTV (and isodose 95\% was set to the prescribed dose) in order to make plan comparisons valid. OARs planning goals included maximizing point doses to the spinal cord and their planned at-risk volumes (3-mm expansion) below their tolerance dose levels, while lowering the endpoint dose to normal liver tissue and right kidney as much as possible. The PTV dose-volume constraints and OARs dose constraints are shown in Table 1. All clinical plans were manually optimized by an expert liver dosimetrist and each IMRT plan met the clinical protocol. Patients exhibited high variability in tumor position and size, as well as OAR exposure. All optimization and dose calculations were performed the dose volume optimization (DVO) version 13.5 .35 and the anisotropic analytical algorithm (AAA) version 13.5.35 with a calculation grid of $2.5 \mathrm{~mm}$. IMRT planning in Eclipse creates highly conformal dose distributions for liver cancer by continuously optimizing the beam intensity modulation to satisfy the institutional optimization protocol. Eclipse IMRT planning combines intensity modulation and inverse planning to accomplish this goal [27].

\section{Model library and DVH estimation model configuration}

The model library consisted of 60 liver cancer patients treated as above. From this library, all 60 patients were selected for Model-G. The average target volume was $147.2 \pm 83.6 \mathrm{~cm}^{3}$ (range: $15.7-298.3 \mathrm{~cm}^{3}$ ). No other specific criteria were applied. For Model-S, 30 patients were selected, with the distance from PTV to the right kidney of less than $3 \mathrm{~cm}$. The average target volume was $155.69 \pm$ $77.5 \mathrm{~cm}^{3}$ (range: $30.4-306 \mathrm{~cm}^{3}$ ). Table 2 shows the volumes of the PTV, liver, L-PTV (normal liver) and right kidney, included in the two models.

RapidPlan was used to create a knowledge-based model that predicts achievable ranges of DVHs for individual OARs of prospective patients. The model libraries contain all planning CTs, structure sets, and dose distributions of previously treated patients. The model configuration

Table 1 Dose-volume endpoint evaluation

\begin{tabular}{ll}
\hline Structures & Acceptable criteria \\
\hline PTV & $V_{95 \%}>95 \%$ \\
& $D_{98 \%}>47.50 \mathrm{~Gy}$ \\
& $D_{2 \%}<55.02 \mathrm{~Gy}$ \\
Normal liver & Dmean $<23 \mathrm{~Gy}$ \\
& $\mathrm{~V}_{30 \mathrm{~Gy}}<28 \%$ \\
& $\mathrm{~V}_{40 \mathrm{~Gy}}<24 \%$ \\
Spinal cord & Dmax $<40 \mathrm{~Gy}$ \\
Right kidney & Dmean $<18 \mathrm{~Gy}$ \\
& $\mathrm{~V}_{5 \mathrm{~Gy}}<70 \%$ \\
& $\mathrm{~V}_{10 \mathrm{~Gy}}<55 \%$ \\
& $\mathrm{~V}_{15 \mathrm{~Gy}}<35 \%$ \\
\hline
\end{tabular}

Abbreviations: PTV planning target volume, Dmean the mean dose for the normal liver or right kidney, Dmax the maximum dose for the spinal cord, $V x G y$ volume receiving at least XGy, $D_{Y \%}$ dose delivered to at least Y\% of the volume 
Table 2 Size of PTV and OAR of patients in Model-G, Model-S and the evaluation group

\begin{tabular}{llllll}
\hline \multicolumn{1}{c}{ Volume } & & & & \\
\hline Group & $(\mathrm{cm})$ & PTV & Liver & L-PTV & Kidney-R \\
Model-G & Mean & $149.2 \pm 83$ & $1580.1 \pm 501.3$ & $1452.8 \pm 480$ & $172.7 \pm 36.4$ \\
& Range & $15.7-298.3$ & $779.2-2992.2$ & $733.1-2905.9$ & $114.7-280$ \\
Model-S & Mean & $158.9 \pm 78$ & $1539.6 \pm 441.7$ & $1430.8 \pm 422.4$ & $176.4 \pm 37.6$ \\
& Range & $30.4-306$ & $779.2-2773.6$ & $733.1-2712.1$ & $115-280$ \\
EG & Mean & $163.1 \pm 73.9$ & $1550.3 \pm 542.5$ & $1354.0 \pm 434.9$ & $184.4 \pm 44.1$ \\
& Range & $24-287.5$ & $822-2896$ & $689.3-2265$ & $122.6-292$ \\
\hline
\end{tabular}

Abbreviations: L-PTV normal liver structures, Kidney-R right kidney structures, EG evaluation group

Data were averaged over their respective patients. The range shows the smallest and largest volume deviations

should conceptually consist of multiple kinds of PTV and OAR geometries and alterations in OAR dosimetry, resulting exclusively from geometric alterations.

During the training, the system analyzed the patient anatomy and DVHs in the plans using principal component analysis (PCA), and created the final mathematical DVH estimation model. Then, the results of the model training were verified using statistical presentations of the training set. Regression, residual and DVH-plots help in estimating the quality of the model and finding potential outlier values that differ from the average in the training set [25]. The outliers must be processed, and after that, the plan data are re-extracted and the model is retrained iteratively until the results are acceptable.

An OAR is designated as an outlier when one or more of these metrics lie outside the range of values found in the model. Using this strategy, 14 of 60 patients in Model-G and 6 of 30 patients in Model-S were identified as containing 1 outlier OAR. RapidPlan requires each OAR to be present in at least 20 plans included in the model. We removed statistical outliers from the training set, rather than deleting the whole plan. Table 3 shows the number of structures included in the two models.

\section{Evaluation group}

An evaluation group consisting of 13 previous patients, treated in 2016, was used to test the RapidPlan results. Patients in the evaluation group were not included in the RapidPlan model libraries. The evaluation group was similar to that included in Model-S, where the distance from the right kidney to PTV was less than $3 \mathrm{~cm}$. In the

Table 3 Number of structures included in Model-G and Model-S

\begin{tabular}{llllllll}
\hline Group & PTV & B-P & Liver & L-PTV & Kidney-R & SC & SC-0.3 \\
\hline Model-G & 59 & 57 & 58 & 58 & 53 & 50 & 52 \\
Model-S & 28 & 28 & 28 & 29 & 25 & 27 & 23 \\
\hline
\end{tabular}

Abbreviations: $B-P$ body-PTV, L-PTV normal liver structures, Kidney- $R$ right kidney structures, $S C$ spinal cord, SC-0.3 spinal cord's planning at-risk volumes (3-mm expansion) evaluation group, the average target volume was $163.1 \pm$ $73.9 \mathrm{~cm}^{3}$ (range: $24-287.5 \mathrm{~cm}^{3}$ ). OARs typically included the liver, L-PTV (normal liver), the spinal-cord and their planned at-risk volumes (3-mm expansion). The volumes of the PTV, liver, L-PTV and right kidney, included in the evaluation group, are shown in Table 2.

The two models were used to generate optimization objectives and automatically optimize treatments for patients in the evaluation group, using the Eclipse treatment planning system. The PTV and OAR objectives are shown in Table 4. Optimization and dose calculation were performed using the Photon Optimization (PO) version 13.5.35 and AAA version 13.5.35. Knowledge-based IMRT plans were created for the evaluation group by Model-G and Model-S, named RPG-plans and RPS-plans, respectively, collectively termed RP-plans. Furthermore, these plans were manually optimized by an expert liver physicist, defined as M-plans.

\section{Evaluating the performance of RapidPlan}

Comparisons of the differences in the DVHs, not only between RP-plans and their respective M-plans, but also between RPG-plans and RPS-plans were performed. RapidPlan results were compared based on target dose coverage and normal tissue sparing. The target dose coverage includes: (1) the homogeneity index (HI)

Table 4 The PTV and OAR optimization objectives for evaluation group

\begin{tabular}{lllll}
\hline Structures & Type & Volume [\%] & Dose & Priority \\
\hline PTV & Upper & 0.0 & 5500 cGy & 500 \\
& Upper & 2.0 & 5400 cGy & 500 \\
& Lower & 100 & 5000 cGy & 500 \\
& Lower & 98 & 5050 cGy & 500 \\
Spinal cord & Upper & 0.0 & 3500 cGy & 150 \\
Right kidney & Line & Generated & Generated & 100 \\
Normal liver & Line & Generated & Generated & 100 \\
\hline
\end{tabular}

Abbreviations: PTV planning target volume 
calculated for PTV using $\left[\mathrm{HI}=\left(\left(\mathrm{D}_{2 \%}-\mathrm{D}_{98 \%}\right) / \mathrm{D}_{\mathrm{p}}\right) \times\right.$ $100 \%]$, where $D_{p}=$ prescribed dose; a lower $H I$ value indicates that the dose coverage is more homogeneous [19]; (2) the conformity index (CI) proposed by Nakamura et al. [29]: $\mathrm{CI}=\mathrm{TV} \times \mathrm{PIV} / \mathrm{TV}_{\mathrm{PIV}}^{2}$, where $\mathrm{TV}=$ target volume, $\mathrm{PIV}=$ prescribed isodose volume, and $\mathrm{TV}_{\mathrm{PIV}}=$ target volume receiving the prescribed dose. The dose coverage and conformity are better when the value of $\mathrm{CI}$ is closer to 1 . The normal tissue sparing is based on statistical average doses to normal liver $\left(D_{\text {mean }}, V_{30 G y}\right.$ $\left.V_{30 \text { Gy }}, V_{40 \text { Gy }}\right)$, spinal-cord $\left(D_{\max }\right)$, and right kidney $\left(D_{\text {mean }}, V_{5 G y}, V_{10 G y}, V_{15 G y}\right)$. Paired $t$-tests were performed to determine significant differences $(p<0.05)$ between
RP-plans and their respective M-plans. The target and normal tissue constraints shown in Table 1 were used to compare all patients in the evaluation group.

\section{Results}

All knowledge-based plans were deemed to conform with the liver IMRT clinical protocol used at our institution, regarding dose-volume constraints. Table 5 and Fig. 1 summarize the RapidPlan results for the evaluation group, averaged over all patients, whereas Fig. 2 shows results for individual patients.

The comparison of RP-plans to M-plans revealed that RP-plans significantly improved $V_{95 \%}$ of PTV (RPG-plans

Table 5 Dosimetric comparison of M-plans, RPG-plans and RPS-plans; data shown are the average of their respective parameters for the 13 patients

\begin{tabular}{|c|c|c|c|c|}
\hline Structures & M-plans & RPG-plans & RPS-plans & $P$ \\
\hline \multirow[t]{2}{*}{ PTV V ${ }_{95 \%}[\%]$} & $97.9 \pm 1.1$ & $98.4 \pm 0.8$ & $98.5 \pm 0.6$ & $\mathrm{i}:<0.05$, ii: $<0.05$ \\
\hline & {$[95.3-99.4]$} & [96.6-99.4] & [97.4-99.4] & iii: NS \\
\hline \multirow[t]{2}{*}{ PTV D ${ }_{98 \%}[\mathrm{~Gy}]$} & $50 \pm 0.5$ & $50.2 \pm 0.5$ & $50.2 \pm 0.3$ & i: NS, ii: NS \\
\hline & {$[48.8-50.8]$} & [49.8-50.9] & {$[49.7-50.6]$} & iii: NS \\
\hline \multirow[t]{2}{*}{ PTV D $2 \%[G y]$} & $54.8 \pm 0.3$ & $54.7 \pm 0.2$ & $54.8 \pm 0.2$ & i: NS, ii: NS \\
\hline & {$[54-55.1]$} & {$[54.4-55]$} & {$[54.4-55.1]$} & iii: NS \\
\hline \multirow[t]{2}{*}{ PTV HI (\%) } & $9.4 \pm 1.4$ & $9.1 \pm 1.2$ & $9.1 \pm 0.9$ & i: NS, ii: NS \\
\hline & {$[8.4-12.8]$} & {$[7.8-11.7]$} & {$[7.4-10.9]$} & iii: NS \\
\hline \multirow[t]{2}{*}{ PTV Cl } & $1.113 \pm 0.037$ & $1.117 \pm 0.039$ & $1.102 \pm 0.05$ & i: NS, ii: NS \\
\hline & [1.06-1.191] & [1.037-1.207] & [1.069-1.177] & iii: $<0.05$ \\
\hline \multirow[t]{2}{*}{ Normal liver Dmean [Gy] } & $10.8 \pm 4$ & $10.8 \pm 4$ & $11.1 \pm 4.2$ & i: NS, ii: < 0.05 \\
\hline & {$[3.7-18.6]$} & [3.7-18.6] & [3.1-18.7] & iii: $<0.05$ \\
\hline \multirow[t]{2}{*}{ Normal liver $V_{20 G y}[\%]$} & $20.1 \pm 11.1$ & $20.1 \pm 10.3$ & $22.6 \pm 12.3$ & i: NS, ii: < 0.05 \\
\hline & {$[4.1-46.3]$} & {$[3.8-43.4]$} & {$[2.6-46.2]$} & iii: $<0.05$ \\
\hline \multirow[t]{2}{*}{ Normal liver $V_{30 G y}[\%]$} & $8.6 \pm 5$ & $9.2 \pm 5.9$ & $10.1 \pm 6.7$ & i: NS, ii: < 0.05 \\
\hline & {$[1.1-19.4]$} & {$[0.9-21.2]$} & {$[0.9-22.8]$} & iii: NS \\
\hline \multirow[t]{2}{*}{ Normal liver V40Gy $_{\text {[\%] }}$} & $2.6 \pm 1.8$ & $2.3 \pm 2$ & $2 \pm 1.4$ & i: NS, ii: < 0.05 \\
\hline & {$[0.1-6.3]$} & {$[0.0-5.7]$} & {$[0.0-4.5]$} & iii: NS \\
\hline \multirow[t]{2}{*}{ Spinal cord Dmax [Gy] } & $19.6 \pm 8.4$ & $20.7 \pm 8.2$ & $19.0 \pm 7.6$ & i: NS, ii: NS \\
\hline & {$[6.2-39.5]$} & {$[7.5-39.3]$} & {$[7.7-34.4]$} & iii: $<0.01$ \\
\hline \multirow[t]{2}{*}{ Right kidney Dmean [Gy] } & $4.5 \pm 2.8$ & $3.9 \pm 2.4$ & $3.8 \pm 2.3$ & $\mathrm{i}:<0.01$, ii: $<0.01$ \\
\hline & {$[0.4-8.7]$} & {$[0.4-7.3]$} & {$[0.4-7.9]$} & iii: NS \\
\hline \multirow[t]{2}{*}{ Right kidney $\vee_{5 G y}[\%]$} & $22.3 \pm 20.4$ & $21.5 \pm 18.8$ & $21.5 \pm 18.6$ & $\mathrm{i}:<0.01$, ii: $<0.01$ \\
\hline & {$[0.1-63.4]$} & {$[0.2-62.3]$} & {$[0.2-63.4]$} & iii: NS \\
\hline \multirow[t]{2}{*}{ Right kidney $V_{10 G y}[\%]$} & $16.9 \pm 13.5$ & $13.8 \pm 10.3$ & $13.3 \pm 10$ & $\mathrm{i}:<0.05$, ii: $<0.05$ \\
\hline & {$[0.0-41]$} & {$[0.0-28]$} & {$[0.0-33.7]$} & iii: NS \\
\hline \multirow[t]{2}{*}{ Right kidney $V_{15 G y}[\%]$} & $10.7 \pm 6.7$ & $8.1 \pm 5.9$ & $7.6 \pm 5.9$ & $\mathrm{i}:<0.01$, ii: $<0.05$ \\
\hline & {$[0.0-18.7]$} & {$[0.0-18.3]$} & {$[0.0-16.6]$} & iii: NS \\
\hline \multirow[t]{2}{*}{ Right kidney $V_{20 G y}[\%]$} & $6.0 \pm 4.5$ & $5.6 \pm 4.6$ & $4.5 \pm 4.0$ & i: NS, ii: NS \\
\hline & {$[0.0-12.8]$} & {$[0.0-13.7]$} & {$[0.0-12.7]$} & iii: $<0.05$ \\
\hline
\end{tabular}

Abbreviations: $\mathrm{HI}$ homogeneity index, Cl conformity index, RPG-plans RapidPlan plans using Model-G, RPS-plans RapidPlan plans using Model-S, NS not significant, $i$ M-plans vs. RPG-plans, ii M-plans vs. RPS-plans, iii RPG-plans vs. RPS-plans. $P \leq .05$ indicates a statistically significant change 

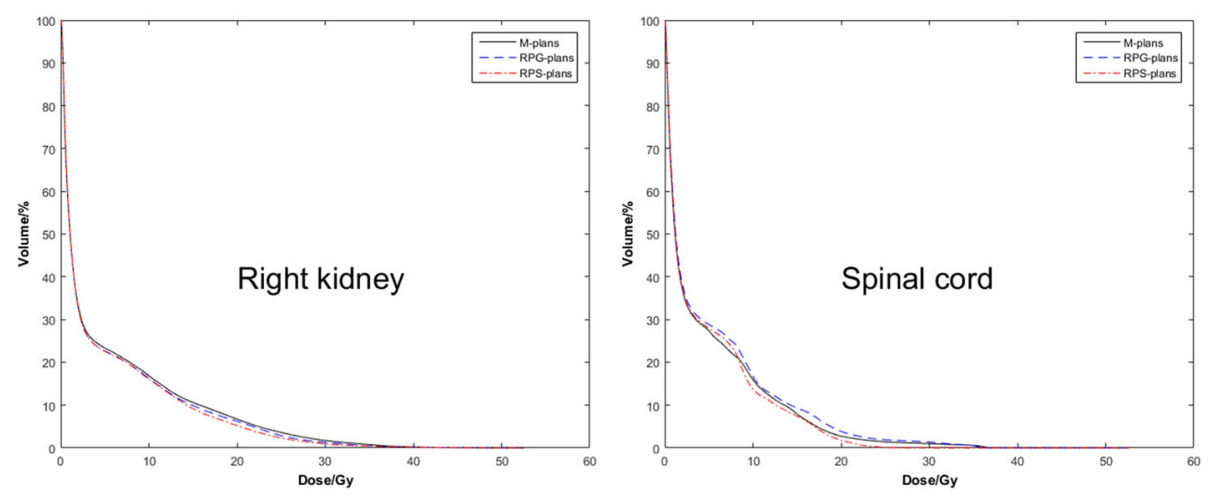

Fig. 1 Average DVHs for the M-plans (solid lines), RPG-plans (dashed lines), RPS-plans (dotted lines) for the evaluation group

vs. RPS-plans vs. M-plans: $98.4 \%$ vs. $98.4 \%$ vs. $97.9 \%)$. The $\mathrm{D}_{98 \%}, \mathrm{D}_{2 \%}, \mathrm{HI}$ and CI values of PTV were improved, although most differences were not significant. For the OAR, RP-plans had significantly more sparing of the right kidney (Dmean: RPG-plans vs. RPS-plans vs. M-plans, 3.9 Gy vs. 3.8 Gy vs. 4.5 Gy). For the normal liver, RPGplans delivered similar doses, while RPS-plans delivered higher doses than those delivered by M-plans (Dmean: RPG-plans vs. RPS-plans vs. M-plans, 10.8 Gy vs. $11.1 \mathrm{~Gy}$ vs. 10.8 Gy). No difference was found between RP-plans and M-plans in the maximum dose to the spinal cord.

With respect to RapidPlan models, there are different. For the PTV coverage, the $\mathrm{V}_{95 \%}, \mathrm{D}_{98 \%}, \mathrm{D}_{2 \%}$, and $\mathrm{HI}$ values were very similar, but RPS-plans had better CI values (1.117 vs. $1.102, p<0.05)$. For OAR doses paring, RPS-plans delivered a lower dose than the maximizing point doses to spinal cord (19.0 Gy vs. 20.7 Gy). Regarding the dose sparing for the right kidney, RPS-plans were better than RPG-plans, especially with $\mathrm{V}_{20 \mathrm{~Gy}}(4.5 \%$ vs. $5.6 \%, p<0.05$ ), although others were very similar. Figure 1 shows the advantages of RPS-plans in the DVH curves for the right kidney and spinal cord. For normal liver, RPS-plans delivered a higher dose (Dmean: 10.8 Gy vs. 11.1 Gy, $p<0.05$; $\mathrm{V}_{20 \mathrm{~Gy}}: 20.1 \%$ vs. $22.6 \%, p<0.05$ ), although all RPS-plans were deemed conform with the liver IMRT clinical protocol. However, for certain individual patients, such as patient 11 (Fig. 2), RPG-plans are better for normal liver sparing.

\section{Discussion}

Fogliata et al. [21] tested RapidPlan for the optimization of RapidArc plans, and generated clinically acceptable plans for hepatocellular cancer radiotherapy. This shows that the model is reliable when no special selection criteria are applied to generate the training, i.e. including all cases, for which the only requirement is to be clinically acceptable. However, liver cancer has high variability in tumor size and position, and the general model (Model-G) may have limited accuracy for special patients. Thus, a specific model
(Model-S) was established in this study, using plans with specific anatomical features for individual patients. For Model-G, Jol et al. [27] demonstrated that 30 plans were sufficient for building a general model. However, in this study, Model-G consisted of 60 patients, to ensure the variety of the data, whereas Model-S consisted of 30 patients, to guarantee high similarity in the geometry of the region of interest. The scope of the minimum reasonable sample size will be addressed in further studies. Nevertheless, Jim et al. [27] observed that more OAR outliers did not necessarily translate into a worse OAR dose. Hussein et al. [24] showed that there were insignificant effects on resulting plan quality when removing dosimetric outliers from the model training set.

In this study, we evaluated the prediction capability of two models (Model-G \& Model-S) in knowledge-based IMRT planning for individual liver cancer patients. Pooled results show that, generally, RapidPlan can improve planning quality and efficiency for liver IMRT, and the prediction ability of the two models with different configurations have a remarkable difference. RP-plans significantly improved the target coverage and the sparing of the right kidney compared to the M-plans. The advantages of some RP-plans compared to the M-plans may be due to challenges in performing an interactive planning of plans that contain many OARs optimally and consistently in a limited number of iterations. Comparison of the two models revealed that Model-S improved the $\mathrm{CI}$ and delivered lower dose to the right kidney $\left(\mathrm{V}_{20 G y}\right)$ and spinal cord, while Model-G delivered lower dose to normal liver tissue. This indicates that the RP-plans are sensitive to the configuration of the model library and the anatomical characteristics of the patient that knowledge-based planning is performed on. The degree of similarity of the cases that make up the model library has a significant effect on the predictive capability of the model.

The selection criteria for establishing the specific model in this study was that the distance from PTV to the right kidney should measure less than $3 \mathrm{~cm}$. We expected to 

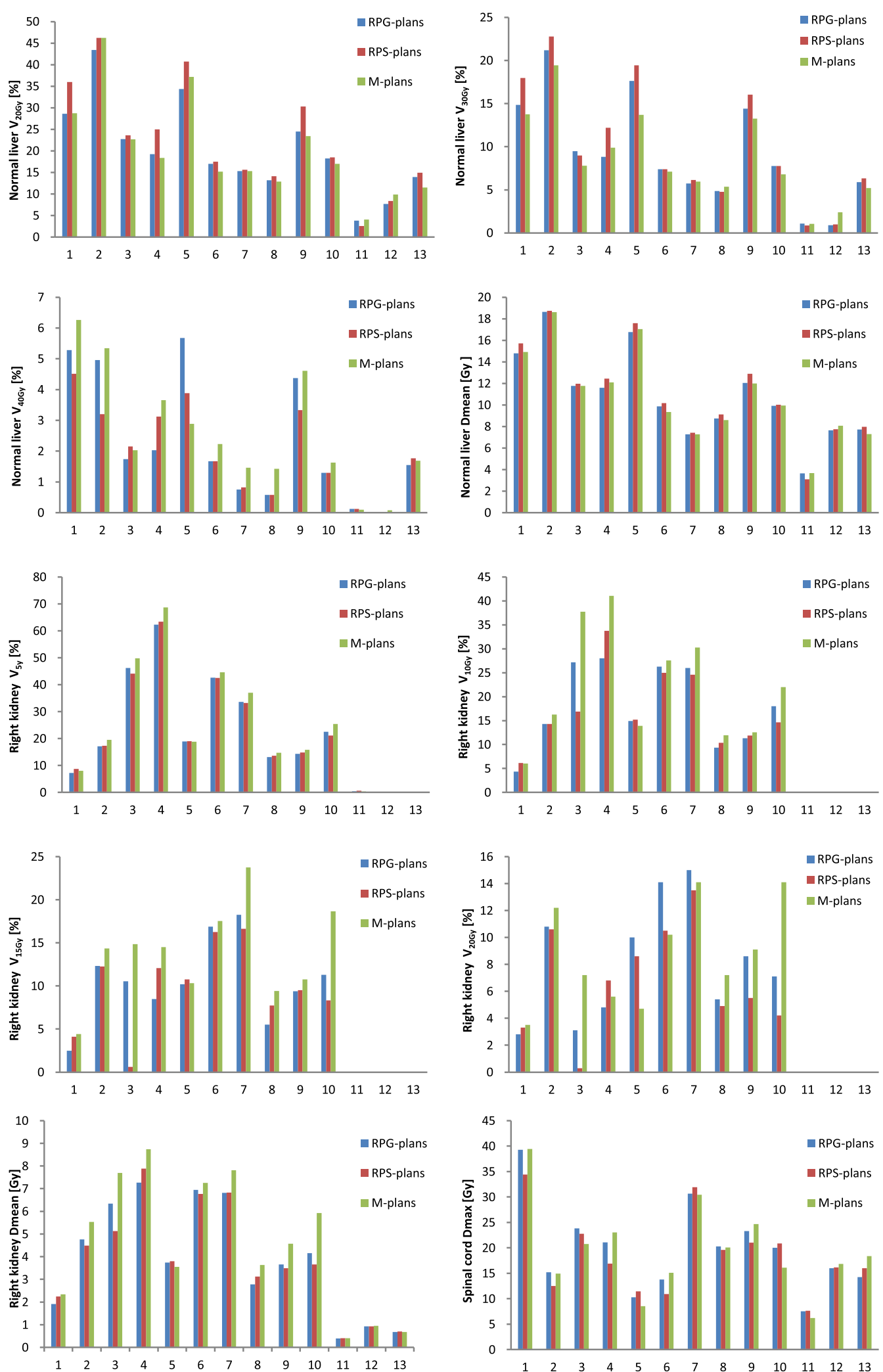

Fig. 2 Histograms show the dosimetric values of respective parameters for the RP-plans and the M-plans of all patients in the evaluation group 
better protect the right kidney, while improving the target coverage, and the experimental results show that Model-S achieved substantial gains compared to Model-G. The pooled data (Table 5 and Fig. 2) shows that RPS-plans slightly improved the right kidney sparing. However, for certain individual patients, RPS-plans had a significant advantage when compared to RPG-plans. For example, in patient 3 (Fig. 1), high variability in right kidney sparing was obtained, RPS-plans decreased $V_{10 G y}, V_{15 G y}, V_{20 G y}$ and Dmean to the right kidney compared to RPG-plans. In patient 11 (Fig. 2), RPG-plans are better for normal liver sparing. From an anatomical point of view, the reason may be that the PTV was small $(24 \mathrm{~cm} 3)$, and located in the inferior segment of the left side of the liver. In addition, it is encouraging that RPS-plans significantly reduced the maximum dose of the spinal cord compared to RPG-plans. This may be due to the fact that the spinal cord has a relatively fixed geometry, adjacent to the kidneys. Therefore, the specific model has great potential in clinical applications for individual patients and we will focus on this.

Some of the findings indicate the different tradeoffs in knowledge-based planning results. According to the pooled results of dosimetry, Model-G is better than the Model-S for normal liver sparing. The following factors may contribute to this: (1) the large size of the liver, only considering the cases with short distance between PTV and the right kidney may not meet the requirements for livers located far away from right kidney; (2) Model-G consisted of more cases, which increases geometric heterogeneity. It is conceivable that further improvements could be made and/or some complex tradeoffs should be addressed. Therefore, some parameters may be adjusted during the optimization process. It is worth noting that a review of plans used for RapidPlan models is still required.

RapidPlan results depend on a variety of factors and the following two are foremost, the geometry of the region of interest and the quality of the plans contained in the model libraries. To ensure the unity of the variables, we built a specific model, which only considers the geometric distance from the PTV to the right kidney, with no restrictions on the other OAR. In addition, one limitation of the study is the small sample size of the evaluation group $(n=13)$. Our study can provide some guidance for clinical applications. Future research will focus on providing optimal allocation of model libraries for individual patients.

\section{Conclusion}

This study shows that RapidPlan can create high quality plans and significantly improve the planning efficiency of IMRT for individual liver cancer patients. Furthermore, these findings demonstrate that the specific model can result in more sparing of OAR, while increasing the conformity index of PTV for liver cancer. Although more systematic studies are needed before a broad clinical application of the proposed methodology, this specific model might be considered as a way to improve the planning quality. Further studies are needed to determine the optimal composition of model libraries, including the relationship between model composition and dosimetry of subsequent plans.

\begin{abstract}
Abbreviations
AAA: Anisotropic analytical algorithm; Cl: Conformity index; DTH: Distance-totarget histograms; DVHs: Dose-volume histograms; DVO: Dose volume optimization; GED: Geometry-based expected dose; HI: Homogeneity index; IMRT: Intensity-modulated radiotherapy; Model-G: General model; Model-S: Specific model; M-plans: Plans were created by manually; OARs: Organs-at-risk; OVH: Overlap volume histogram; PCA: Principal component analysis; PO: Photon optimization; PTV: Planning target volume; RPG-plans: Plans were created by Model-G; RPSplans: Plans were created by Model-S; VMAT: Volumetric modulated arc therapy
\end{abstract}

\section{Acknowledgements}

We want to thank Dr. Lei Xing and Dr. Yong Yang (Department of Radiation Oncology, Stanford University School of Medicine, Stanford, CA, USA) for the fruitful discussions of knowledge-based models and optimization.

\section{Funding}

This work was jointly supported by National Natural Science Foundation of China (61471226, 81530060), Natural Science Foundation for Distinguished Young Scholars of Shandong Province (JQ201516), Natural Science Foundation of Shandong Province (ZR2017BF005). We also thanks to the supporting of Taishan scholar project of Shandong Province (NO.tsqn20161023).

\section{Availability of data and materials}

All data generated or analysed during this study are included in this published article.

\section{Authors' contributions}

$\mathrm{DL}, \mathrm{GY}$ and $\mathrm{YL}$ designed the study and the analysis. DL, GY, $\mathrm{YL}, \mathrm{ZF}, \mathrm{CT}$ and $\mathrm{BL}$ developed the DVH estimation model. YL and ZY contributed to the data analysis. GY and YL designed the study and composed the manuscript. All authors read and approved the final manuscript.

Ethics approval and consent to participate

Not applicable.

\section{Consent for publication}

Written informed consent was obtained from the patient for the publication of this report.

\section{Competing interests}

The authors declare that they have no competing interests.

\section{Publisher's Note}

Springer Nature remains neutral with regard to jurisdictional claims in published maps and institutional affiliations.

Received: 12 November 2017 Accepted: 9 March 2018 Published online: 27 March 2018

References

1. Kudo M. Surveillance, diagnosis, treatment, and outcome of liver cancer in Japan. Liver Cancer. 2015;4:39-50.

2. Djajaputra D, Wu Q, Wu Y, Mohan R. Algorithm and performance of a clinical IMRT beam-angle optimization system. Phys Med Biol. 2003:48:3191.

3. Nelms BE, Robinson G, Markham J, Velasco K, Boyd S, Narayan S, et al. Variation in external beam treatment plan quality: an inter-institutional study of planners and planning systems. Pract Radiat Oncol. 2012;2:296-305.

4. Das IJ, Cheng C-W, Chopra KL, Mitra RK, Srivastava SP, Glatstein E. Intensity-modulated radiation therapy dose prescription, recording, and delivery: patterns of variability among institutions and treatment planning systems. J Natl Cancer I. 2008;100:300-7. 
5. Voet PW, Dirkx ML, Breedveld S, Fransen D, Levendag PC, Heijmen BJ. Toward fully automated multicriterial plan generation: a prospective clinical study. Int J Radiat Oncol Biol Phys. 2013;85:866-72.

6. Hong TS, Craft DL, Carlsson F, Bortfeld TR. Multicriteria optimization in intensity-modulated radiation therapy treatment planning for locally advanced cancer of the pancreatic head. Int J Radiat Oncol Biol Phys. 2008; 72:1208-14.

7. Craft DL, Hong TS, Shih HA, Bortfeld TR. Improved planning time and plan quality through multicriteria optimization for intensity-modulated radiotherapy. Int J Radiat Oncol Biol Phys. 2012;82:e83-90.

8. Chen $\mathrm{H}$, Craft DL, Gierga DP. Multicriteria optimization informed VMAT planning. Med Dosim. 2014;39:64-73.

9. Wu B, Pang D, Simari P, Taylor R, Sanguineti G, McNutt T. Using overlap volume histogram and IMRT plan data to guide and automate VMAT planning: a head-and-neck case study. Med Phys. 2013;40:021714

10. Breedveld S, Storchi PR, Voet PW, Heijmen BJ. iCycle: integrated, multicriterial beam angle, and profile optimization for generation of coplanar and noncoplanar IMRT plans. Med Phys. 2012:39:951-63.

11. Yang Y, Ford EC, Wu B, Pinkawa M, Van Triest B, Campbell P, et al. An overlap-volume-histogram based method for rectal dose prediction and automated treatment planning in the external beam prostate radiotherapy following hydrogel injection. Med Phys. 2013;40:011709.

12. Wu B, McNutt T, Zahurak M, Simari P, Pang D, Taylor R, et al. Fully automated simultaneous integrated boosted-intensity modulated radiation therapy treatment planning is feasible for head-and-neck cancer: a prospective clinical study. Int J Radiat Oncol Biol Phys. 2012;84:e647-e53.

13. Lian J, Yuan L, Ge Y, Chera BS, Yoo DP, Chang S, et al. Modeling the dosimetry of organ-at-risk in head and neck IMRT planning: an intertechnique and interinstitutional study. Med Phys. 2013;40:121704.

14. Liu BJ. A knowledge-based imaging informatics approach for managing proton beam therapy of cancer patients. Technol CANCER Res T. 2007;6:77-84.

15. Lee KJ, Barber DC, Walton L. Automated gamma knife radiosurgery treatment planning with image registration, data-mining, and Nelder-mead simplex optimization. Med Phys. 2006;33:2532-40.

16. Wu B, Ricchetti F, Sanguineti G, Kazhdan M, Simari P, Chuang M, et al. Patient geometry-driven information retrieval for IMRT treatment plan quality control. Med Phys. 2009;36:5497-505.

17. Wu B, Ricchetti F, Sanguineti G, Kazhdan M, Simari $P$, Jacques $R$, et al. Datadriven approach to generating achievable dose-volume histogram objectives in intensity-modulated radiotherapy planning. Int J Radiat Oncol Biol Phys. 2011;79:1241-7.

18. Chanyavanich V, Das SK, Lee WR, Lo JY. Knowledge-based IMRT treatment planning for prostate cancer. Med Phys. 2011;38:2515-22.

19. Good D, Lo J, Lee WR, Wu QJ, Yin F-F, Das SK. A knowledge-based approach to improving and homogenizing intensity modulated radiation therapy planning quality among treatment centers: an example application to prostate cancer planning. Int J Radiat Oncol Biol Phys. 2013;87:176-81.

20. Zhu X, Ge Y, Li T, Thongphiew D, Yin F-F, Wu QJ. A planning quality evaluation tool for prostate adaptive IMRT based on machine learning. Med Phys. 2011;38:719-26.

21. Fogliata A, Wang P-M, Belosi F, Clivio A, Nicolini G, Vanetti E, et al. Assessment of a model based optimization engine for volumetric modulated arc therapy for patients with advanced hepatocellular cancer. Radiat Oncol. 2014;9:236-48.

22. Fogliata A, Belosi F, Clivio A, Navarria P, Nicolini G, Scorsetti M, et al. On the pre-clinical validation of a commercial model-based optimisation engine: application to volumetric modulated arc therapy for patients with lung or prostate cancer. Radiother Oncol. 2014;113:385-91.

23. Wu H, Jiang F, Yue H, Zhang H, Wang K, Zhang Y. Applying a RapidPlan model trained on a technique and orientation to another: a feasibility and dosimetric evaluation. Radiat Oncol. 2016;11:108.

24. Hussein M, South CP, Barry MA, Adams EJ, Jordan TJ, Stewart AJ, et al. Clinical validation and benchmarking of knowledge-based IMRT and VMAT treatment planning in pelvic anatomy. Radiother Oncol. 2016;120:473-9.

25. Chang AT, Hung AW, Cheung FW, Lee MC, Chan OS, Philips H, et al. Comparison of planning quality and efficiency between conventional and knowledge-based algorithms in nasopharyngeal Cancer patients using intensity modulated radiation therapy. Int J Radiat Oncol Biol Phys. 2016;95:981-90.

26. Berry SL, Ma R, Boczkowski A, Jackson A, Zhang P, Hunt M. Evaluating inter-campus plan consistency using a knowledge based planning model. Radiother Oncol. 2016;120:349-55.
27. Tol JP, Delaney AR, Dahele M, Slotman BJ, Verbakel WF. Evaluation of a knowledge-based planning solution for head and neck cancer. Int J Radiat Oncol Biol Phys. 2015;91:612-20.

28. Delaney AR, Tol JP, Dahele M, Cuijpers J, Slotman BJ, Verbakel WF. Effect of dosimetric outliers on the performance of a commercial knowledge-based planning solution. Int J Radiat Oncol Biol Phys. 2016;94:469-77.

29. Nakamura JL, Verhey LJ, Smith V, Petti PL, Lamborn KR, Larson DA, et al. Dose conformity of gamma knife radiosurgery and risk factors for complications. Int J Radiat Oncol Biol Phys. 2001;51:1313-9.

\section{Submit your next manuscript to BioMed Central and we will help you at every step:}

- We accept pre-submission inquiries

- Our selector tool helps you to find the most relevant journal

- We provide round the clock customer support

- Convenient online submission

- Thorough peer review

- Inclusion in PubMed and all major indexing services

- Maximum visibility for your research

Submit your manuscript at www.biomedcentral.com/submit
Biomed Central 